\title{
INJECT AN ELASTIC GRID COMPUTING TECHNIQUES TO OPTIMAL RESOURCE MANAGEMENT TECHNIQUE OPERATIONS
}

\author{
${ }^{1}$ R. Surendran and ${ }^{2}$ B. Parvatha Varthini \\ ${ }^{1}$ Department of Information Technology, Sathyabama University, Chennai, India \\ ${ }^{2}$ Department of Computer Applications, St. Joseph's College of Engineering, Chennai, India
}

Received 2013-06-22, Revised 2013-06-30; Accepted 2013-07-09

\begin{abstract}
Evaluation of sharing on the Internet well- developed from energetic technique of grid computing. Dynamic Grid Computing is Resource sharing in large level high performance computing networks at worldwide. Existing systems have a Limited innovation for resource management process. In proposed work, Grid Computing is an Internet based computing for Optimal Resource Management Technique Operations (ORMTO). ORMTO are Elastic scheduling algorithm, finding the Best Grid node for a task prediction, Fault tolerance resource selection, Perfect resource co-allocation, Grid balanced Resource matchmaking and Agent based grid service, wireless mobility resource access. Survey the various resource management techniques based on the performance measurement factors like time complexity, Space complexity and Energy complexity find the ORMTO with Grid computing. Objectives of ORMTO will provide an efficient Resource co-allocation automatically for a user who is submitting the job without grid knowledge, design a Grid service (portal) for selects the Best Fault tolerant Resource for a given task in a fast, secure and efficient manner and provide an Enhanced grid balancing system for multi-tasking via Hybrid topology based Grid Ranking. Best Quality of Service (QOS) parameters are important role in all RMT. Proposed system ORMTO use the greater number of QOS Parameters for better enhancement of existing RMT. In proposed system, follow the enhanced techniques and algorithms use to improve the Grid based ORMTO.
\end{abstract}

Keywords: Optimal Resource Management Technique Operations, Elastic Scheduling, Best and Fault Tolerance Grid Node Selection, Resource Co-Allocation, Grid Balanced Resource Matchmaking and Mobility Resource Access

\section{INTRODUCTION}

Role of Optimal Resource Management Technique (ORMT) is Request Handle, Dispatch, Schedule, Information Manage, Transfer Manage, Query Process, resource allocation, Broker Resource Management Technique (BRMT), Agent based grid service, resource match making, resource selection, resource discovery, resource coordination, resource monitoring, resource access, resource sharing, resource recovery, resource management, resource access based on broker/agent, resource reservation and utilization, grid balancing. Grid end user or Authorized Resource Requestor asks the Grid service to ORMT. ORMT receive and resolve the request from the grid end user. After the request is resolved, ORMT asks the grid service from the service or resource provider. Here, Resources are services, software, platform, infrastructure, supercomputer, DB, storage space, virtual organization, virtual machine, cluster, server, personal computer, webcam, printer, scanner .... Registered service providers give a response to ORMT. ORMT give response to requester based on high-level scheduling, allocation, monitoring and QOS -Quality of Service (Barzegar et al., 2011)

Corresponding Author: R. Surendran, Department of Information Technology, Sathyabama University, Chennai, India 


\section{PROBLEM STATEMENT}

\subsection{Objectives of Proposed System}

Objectives of ORMTO will provide an efficient Resource co-allocation automatically for a user who is submitting the job without grid knowledge, design a Grid service (portal) for selects the Best Fault tolerant Resource for a given task in a fast, secure and efficient manner and provide an Enhanced grid balancing system for multi-tasking via Hybrid topology based Grid Ranking. End User doesn't want the grid and resource knowledge only submit job to the portal. Grid service (portal) will take care of all knowledge about the Optimal Resource Management Techniques (ORMT) automatically based on OGSA (Open Grid Services Architecture).

\subsection{Issues in Existing System}

- Issue 1: In the Existing system grid technologies do not have efficient resource monitoring and prediction strategies (Ostberg and Elmroth, 2013). It does not co-operate with various components to achieve high performance reason is, it does not have sufficient resources. Finding a Best Grid Node for the Task allocation is the major problem which most of the Grid Infrastructure has in today's world

- Issue 2: Topological structure is not well defined for Fault tolerant Cloud resource selection service

- Issue 3: Scheduling phase is not follow the dynamic and intelligent Grid/Cloud component selection. Scheduling is based on the resource coallocation request from user run-time estimation. As user run-time estimations are usually very imprecise that is not clear

- Issue 4: Need to improve the accuracy and improve the Customer Satisfaction.

- Issue 5: Dynamic cloud load balancing and Fault tolerances technique service time is not good

- Issue 6: Manual fault calculation and Lacking in wireless mobility grid resource access

\subsection{Proposed System Solutions}

It focuses on ORMT:

- Solution 1. Scheduling the resource co-allocation request based on system generated predictions through Discovery service and Priority based on fairness with user history
- Solution 2. Selection of the best Grid node for task prediction in the dynamic grid computing to provide the best Resource selection for jobs and hence yielding the best result based on GRP-PSO

- Solution 3. Faults tolerant grid resource Selection gets high resource Availability based on Hybrid GRP-PSO

- Solution 4. EC2 algorithm use to design a welldefined hybrid topology to increase the quality of grid balancing and perfect matchmaking process

- Solution 5. Broker/Agent Resource Management Techniques is used to increase the speed of Grid Service (Javanmardi et al., 2013) Resource availability is increased from agent migration and fault tolerance agents

- Solution 6. Fault can be identified and load can be balanced very quickly. This fully automated failure Detection Grid service access from anywhere at any time through grid portal

\section{PROPOSED SYSTEM: ORMTO}

Optimal Resource management Technique Operations (ORMTO) Umbrella is covering a more well defined technical components like listed in sub heading (Surendran and Varthini, 2012).

\subsection{Elastic Scheduling Algorithm}

An Elastic Scheduling based on Priority, System history, Grid Network capability, number of jobs with size, system generated prediction and Negotiation. In Existing Model, End User wants the grid and resource knowledge then only possible to work in the grid network (Pradoa et al., 2012). Scheduling is a key issue which must be solved in grid computing study and a better scheduling scheme can greatly improve the efficiency (Raj and Vasudevan, 2011).

\subsubsection{ORMT Based Elastic Scheduling}

In Fig. 1, Scheduling algorithm will also consider various factors like Priority, System history, Grid Network capability, number of jobs with size, system generated prediction, Negotiation and Co-scheduling.

Architecture of the elastic scheduling, starting with the creation of Directed Acyclic Graph, the nodes are being extracted with the corresponding information and can compute the communication demands, host capacity, cost constraint using the fuzzy scheduler, which can be able to determine the starting time of the task and data transfer time. 


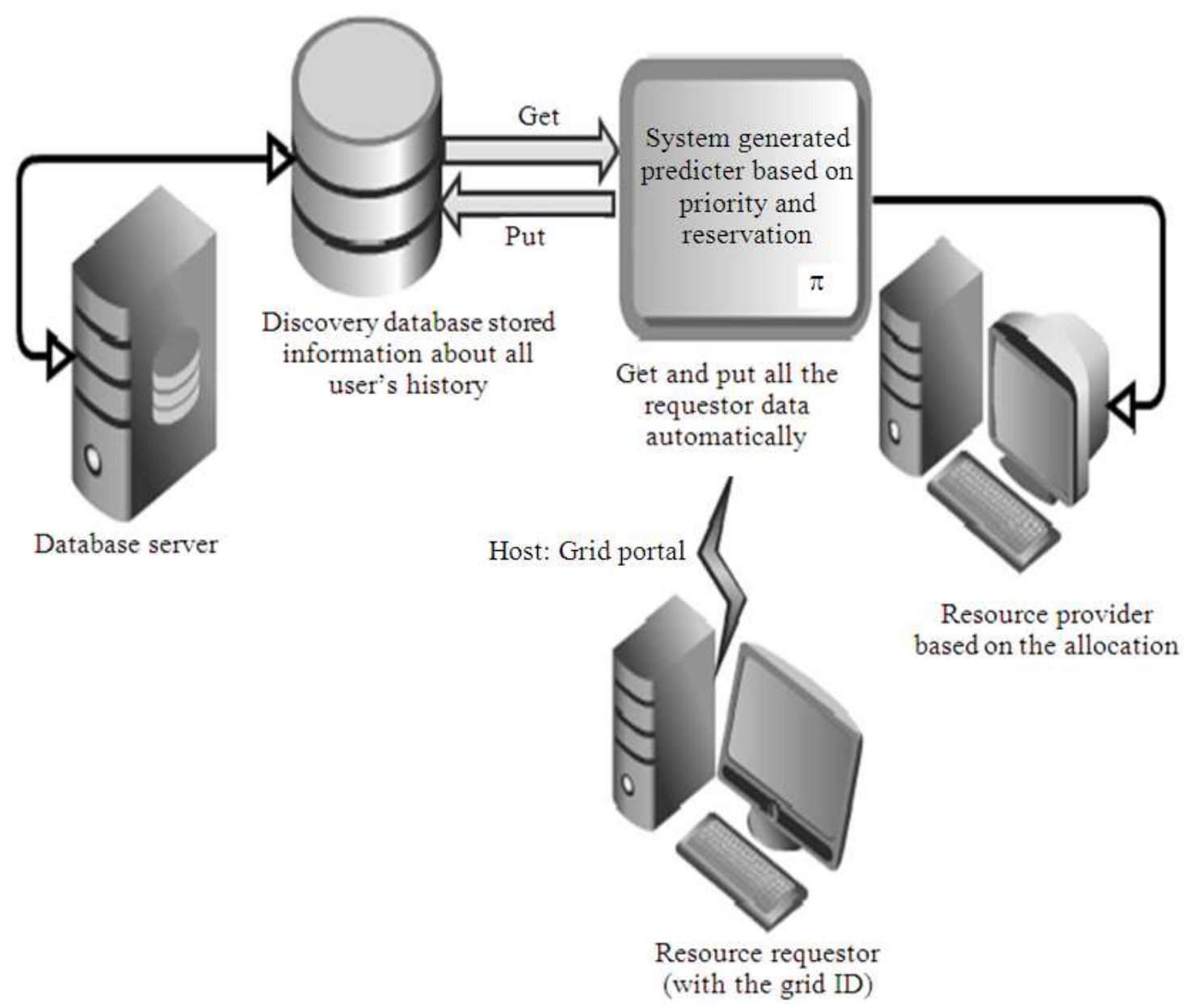

Fig. 1. Block Diagram of ORMT based elastic scheduling

\subsubsection{Algorithm of Elastic Scheduling}

\section{PROCEDURE PRORITY SCHEDULING $(\mathrm{G}, \mathrm{U}, \mathrm{V})$}

//G-Grid network represent by graph

Step 1: Compute the indegree 0 vertex (V) [available free Resource]

Step 2: Store that indegree 0 vertex from priority queue (enqueue and dequeue)

Step 3: Get the next vertex (U) from the grid network

Step 4: For all edges $(\mathrm{U}, \mathrm{V})$ update the indegree of $\mathrm{V}$ and put $\mathrm{V}$ in the queue if the updated indegree 0 .

Repeat steps 3 and 4 while the queue is not empty.

\subsection{Finding the Best Grid Node for a Task Prediction}

Find the Best Grid Node based on GRP (Grid Resource Prediction pattern)-PSO (Particle Swarm
Optimization) Algorithm. Optimization algorithm presented and named as GRP-PSO. GRP- PSO is combination of Particle Swarm optimization (PSO) algorithm and Grid Resource Prediction Pattern (GRP). Present a new optimization algorithm called Genetic Algorithm and show its effectiveness. In existing work, Particle Swarm Optimization algorithm was weak in Local search, Grid Resource Prediction pattern has been used to improve the quality and finding best grid node (Hu et al., 2012).

\subsubsection{ORMT Based Finding the Best Grid Node}

Grid Resource Prediction (GRP) Service sends request to Grid History and Prediction database to update the Grid Node's process and execution of task and also sends request to all the Grid Node to find the best 
Iteration of Resource and task process through the Grid Resource Prediction Patter and Particle Swarm Algorithm. Administrator with multiple or Grid Remote Evaluator service can be same Geographical location or Different Geographical Location. Grid Resource Revelation pattern has History, Grid Resource Revelation Model, Prediction for each Resource (rs). Whenever want to update any data about any of the Grid Node specify it in History Data. Query the history database to find the very least used Resource.

\subsubsection{Algorithm Description}

Step 1: Represent the problem domain as a chromosome of fixed length $\mathrm{n}$; Choose the size of population of chromosomes $\mathrm{N}$, a crossover probability pc and a mutation probability pm

Step 2: Define a fitness function $\mathrm{f}$ to measure the performance (fitness) of an individual chromosome in the problem domain.

Step 3: Randomly generate an initial population of chromosomes of size $\mathrm{N}$ : $\mathrm{x} 1, \mathrm{x} 2, \ldots, \mathrm{xN}$

Step 4: Calculate the fitness of each individual chromosome: $f(x 1), f(x 2), \ldots, f(x N)$

Step 5: Select a pair of chromosomes for mating from the current population. Parent chromosomes are selected with a probability related to their fitness.

Step 6: From those parents, create a pair of offspring chromosomes by applying the genetic operator crossover and mutation.

Step 7: Place the created offspring chromosomes in the new population.

Step 8: Repeat from Step 5 until the new population size equals the old population size

Step 9: Replace the initial chromosome population with the new population

Step 10: Go to Step 4 and repeat the process until some termination (or optimization) criterion is satisfied

\subsubsection{Genetic GRP-PSO Algorithm}

Calculation for Cost evaluation for Grid Computing Resource

Step 1: Start and Initialize every Particle with Random Position and velocity vectors.

// Init a,b with random position in $[-3,3]$ :

double a $=$ Random.NextDouble ()$* 6-3$;

double $\mathrm{b}=$ Random.NextDouble ()$* 6$ - 3;

double[] particlePos $=\{\mathrm{a}, \mathrm{b}\}$;

//Init va, vb Random velocity in $[-3,3]$ :

double va $=$ Random.NextDouble ()$* 6-3$; double $\mathrm{vb}=$ Random.NextDouble ()$* 6-3$; double[] particleVel $=\{\mathrm{va}, \mathrm{vb}\}$;

Step 2: Start finding the cost based on Each Particle using Random values like a,b.

Calculate Cost by passing a, b Param Value and return the cost from Random values $a, b$.

calculateCost(double a, double b)

return $100 *(a * b-b * b) *(a * a-b * b)+(1-a) *(1-a)$ \}

public double CalculateCost1(double a, double y)

$\{$ double $\mathrm{c}=$

$3 *(1-a) *(1-a) * \quad \operatorname{Math} \operatorname{Exp}(-a * a)-(b+1) *(b$ $+1)-10 *(\mathrm{a} / 5-\mathrm{a} * \mathrm{a} * \mathrm{a}-\mathrm{b} * \mathrm{~b} * \mathrm{~b} * \mathrm{~b} * \mathrm{~b}) *$ $\operatorname{Math} \cdot \operatorname{Exp}(-\mathrm{a} * \mathrm{a}-\mathrm{b} * \mathrm{~b})-1 / 3 * \operatorname{Math} \operatorname{Exp}(-(\mathrm{a}+1) *(\mathrm{a}+$ 1) $-\mathrm{b} * \mathrm{~b})$

return $1-\mathrm{c}$;

Step 3: Use the history data and Grid Resource Prediction Pattern Data (GRP) from the Prediction database to find the pbest value.

Step 4: Match this Current Cost (C) with Best value so far (pbest), When Cost (C) is better than pbest value assign it pbest using Cost(c).

For each Particle Position Evaluate $\operatorname{cost}(\mathrm{C})$

If Cost (C) is better than pbest then

$$
\text { End if }
$$

Loop

Step 5: Result of pbest will be considered to be lowest cost of all particles. Assign the pbest lowest cost value to gbest.

Step 6. . Gbest $=$ pbest

NewVel = New Velocity

CurVel = Current Velocity

Ran1, Ran2 = Random Number in the interval

$$
\begin{aligned}
& \text { Coeff } 1 \text {, Coeff } 2=\text { Accelerated Coefficient } \\
& \text { CurPos= Current Position } \\
& \text { NewPos= New Position }
\end{aligned}
$$

Calculate the New velocity using below formula

NewVel = CurVel + Coeff1.ran1. (pbest-a) + Coeff2.ran2. (gbest - a)

Step 7: Calculate the New Position using below formula NewPos= CurPos+NewVel

Iterate the about formula to find the best position with Minimum cost value.

\subsubsection{Experimental Result of Best Grid node Detection}

Best Grid Nodes found from the values of shortest distance, population, individual, fittest, genome by using Genetic Algorithm. 
R. Surendran and B. Parvatha Varthini / Journal of Computer Science 9 (8): 1051-1060, 201

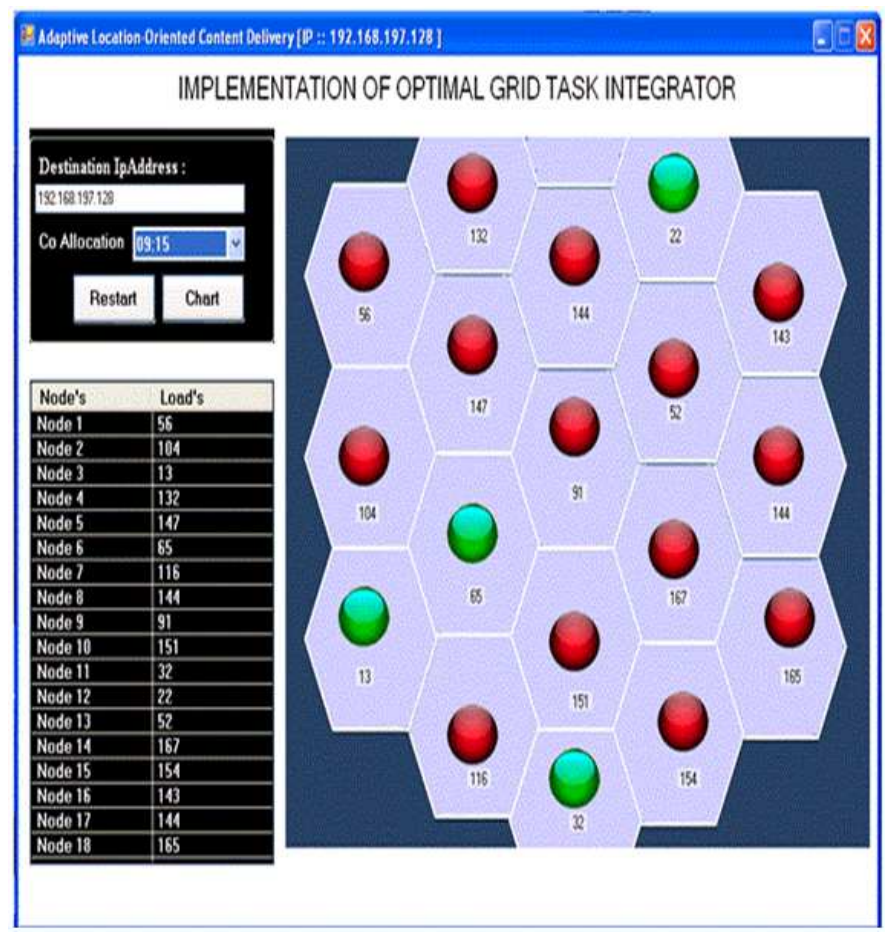

Fig. 2. Job runs through best grid nodes

Implementation of optimal grid task integrator

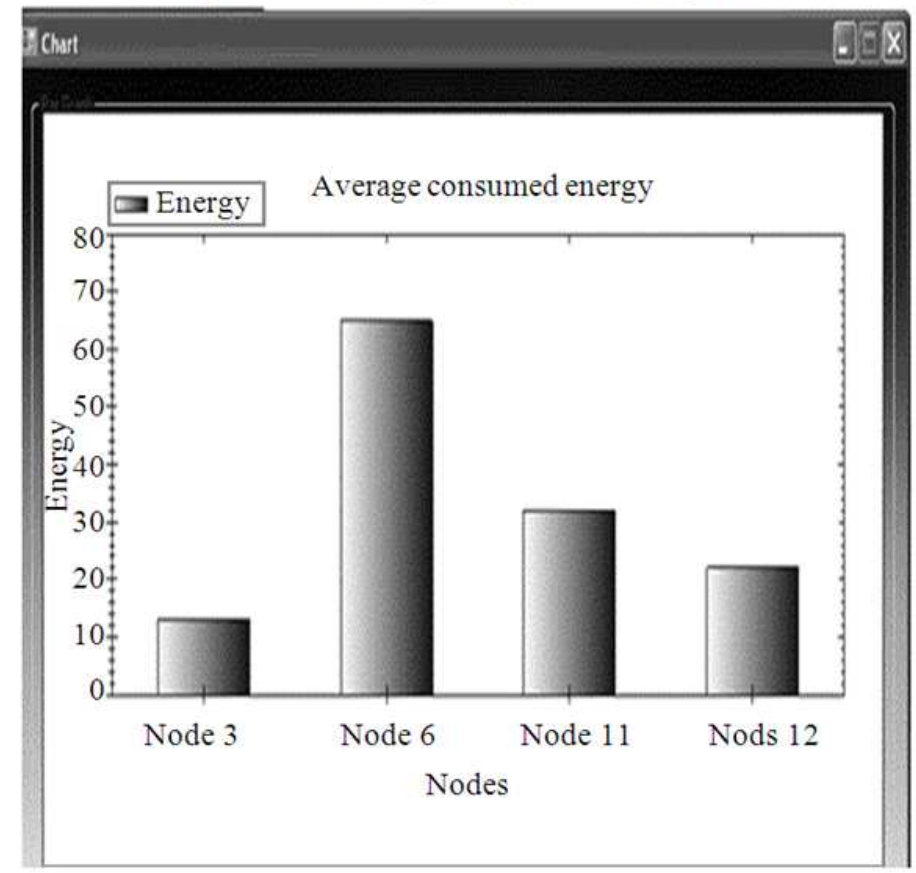

Fig. 3. Energy level chart for best grid nodes 


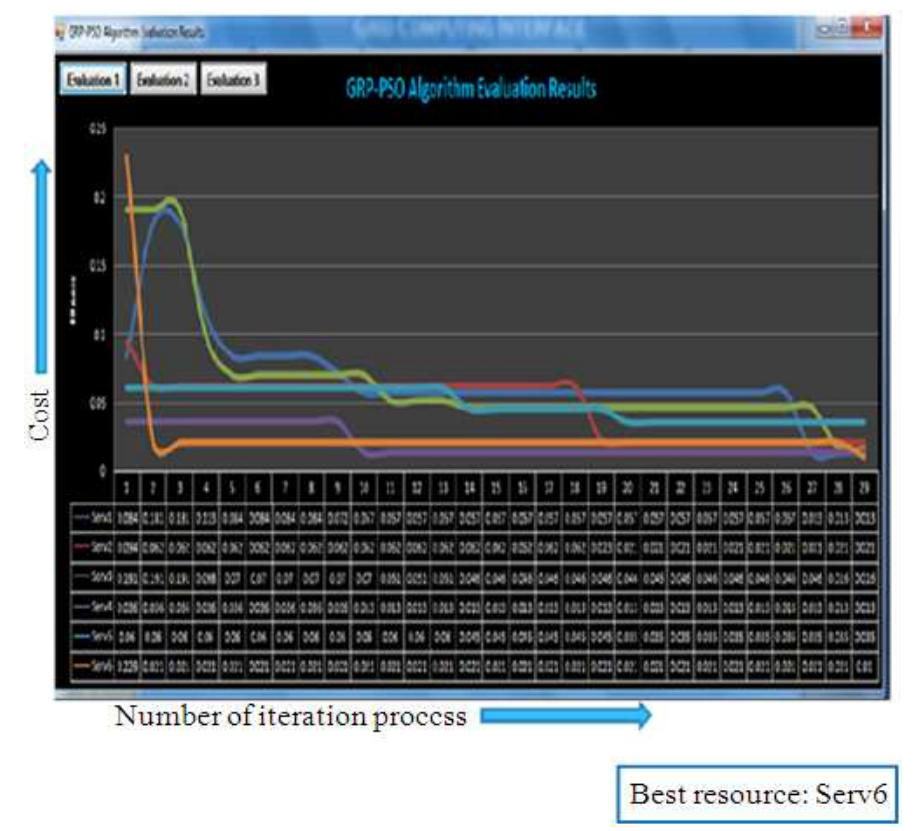

Fig. 4. Hybrid Genetic GRP-PSO based faults tolerant resource selection

Figure 2, shows the Job execution flow through Best Grid Nodes. For Best Resources Monitoring we take one sample application as File Transfer Grid Service with energy level from Fig. 3.

\subsection{Fault Tolerance Resource Selection}

Perfect fault tolerant grid resource selection for multi task prediction is based on Hybrid Genetic GRP-PSO algorithm. Grid Computing has limitations in the existing system for resource monitoring and prediction strategies (Bouyer and Sap, 2010). This grid technology does not have efficient resource monitoring and prediction strategies (Dobre et al., 2011).

\subsubsection{ORMT Based for Fault Tolerance Resource Selection}

Based on Single Sign on, Hybrid Genetic GRP- PSO is combination of Particle Swarm optimization (PSO) algorithm and Grid Resource Prediction Pattern (GRP) from two or more grid networks.

\subsubsection{Algorithm Description}

Faults Tolerant Grid Resource Selection ()

Input: Set of available resources $\{\mathrm{R}\}$

Output: Resource selection from requirements

Step 1: Detect the possible faults at the time of resource selection.
Step 2: Design and implement a fault detector and a fault manager.

Step 3: Compute the Resource based on high ranking, high priority, failure aware, less bandwidth

Fault tolerances grid system gets high resource Availability.

\subsubsection{Faults Tolerant Resource Selection Results}

In Fig. 4, Minimum Cost value can be achieved through above iteration using Hybrid Genetic GRP-PSO Algorithm. Finding the Minimum cost value to find the best node in grid Environment is found through the Iterated Minimum cost value.

\subsection{Perfect Resource Co-Allocation}

Enhanced Resources Co-Allocation are used to allocate the multiple resources for different applications efficiently. Different resources are not available in an every workplace that time co-Allocation help to access the all resources from anywhere at any time via grid computing. In Existing Model, scheduling the resource co-allocation request is done using user run time estimation. As user run time estimations are usually very imprecise that is not clear (Netto and Buyya, 2008).

\subsubsection{ORMT based for Resource co-Allocation}

System generated predictions are better parameters than user run time estimates for Resource co-Allocation (Netto et al., 2011). 


\subsubsection{Algorithm}

\section{SYSTEM GENERATED PREDICTION ( )}

Step 1: Aggregate the job have executed in the past based on similarity jobs executed on grid network (user History and utilization of resource)

Step 2: Predict from technicality, usability issues, priority And Misconceptions

Step 3: Predict the code analysis, analytic benchmarking/Code profiling and statistical prediction

\subsubsection{Partitioning Leach Cluster's Algorithm Description}

Step 1: Select no of resource requester placed in dynamic approach.

Step 2: Submission of user query to resource broker.

Step 3: Sent user's job to resource provider, resource provider process the job request.

Step 4: To find tower based on shortest energy path approach based on leach cluster mechanism.

Step 5: To correlate tower position $(\mathrm{x}, \mathrm{y})$ with mobility node position(x,y) for partitioning based clustering technique used to find shortest energy path.

Step 6: To find shortest energy path, we have taken Number of mobility nodes Vs tower position using energy optimization technique.

\subsection{Grid Balanced Resource Matchmaking}

Grid balanced Resource matchmaking based on Evaluation Come Classification (EC2) to a Job. Grid balanced Resource matchmaking process achieve Coordinated and optimized resource sharing, enhanced security management, Cost optimizations. Improved performance substantially, backup plan in case the system fails even partially, maintain the system stability, accommodate future modification in the system and avoid a situation where some nodes are heavily loaded while others are idle or doing little work. Most Resource matchmaking approaches are depend on the best grid resource selection from fault tolerance, shortest distance, low cost, high energy, load balanced techniques (Qiang et al., 2011; Ning and Shaohua, 2013; Folling et al., 2010). Existing systems have a Limited innovation for resource matchmaking process.

Optimal RMT for Grid balanced Resource grid balanced resource selection based on Evaluation Come Classification (EC2) to a job at Runtime. EC2 algorithm describes three things such as:

\section{- Grid resource evaluation and classification}

Evaluation: Based on Resource Discovery, Resource Monitoring and QoS (Quality of Service) parameters.

Classification: Grid ranking use to classify the grid resources based on QoS parameters:

\section{- Grid Evaluation system by Scheduler}

Scheduler rating the resources based on grid ranking. Grid ranking provide a high priority rating for local scheduler (no need of network bandwidth and transport time), Next medium priority rating for Meta scheduler (advanced reservation) and then next low priority rating for remote scheduler.

Scheduler evaluates the job based on the following steps, Step 1: Job Submission; Step 2: Job Monitoring; Step 3: Job Control; Step 4: Job Migration; Step 5: Job Termination.

3. Job evaluation and classification. EC2 algorithm use to design a well- defined hybrid topology, Increase the quality of grid balancing and Detect the fault tolerance grid resource from the available resources with an advanced concept of resource co-allocation, Perfect priority scheduling, Grid ranking, resource communication intensive and Grid balancing:

- Case 1: Based on job execution time

- Case 2: Based on priority value of job

- Case 3: Based on environmental status of job

- Case 4: Based on situation base of job processing

- Case 5: Based on job similarity

- Case 6: Based on job's working platform

\subsection{Agent Based Grid Service and BRMT}

Large Scale Multi Agent's based Resource Management on grid Service. Agents are used to migrate, one supplier to another supplier easily based on grid balancing technique. Large scale agent means number of agents is large. For deploying the application via host, first predict the best agent from a lot of distributed active agents (Surendran and Varthini, 2012). Resource availability is increased from Broker/agent migration and fault tolerance agents. Richer forms of sharing and increased qualities of service in Agent based grid service.

\subsubsection{Broker's Resource Management Technique (BRMT)}

The resource broker provides pairing services between the service requester and the service provider. 


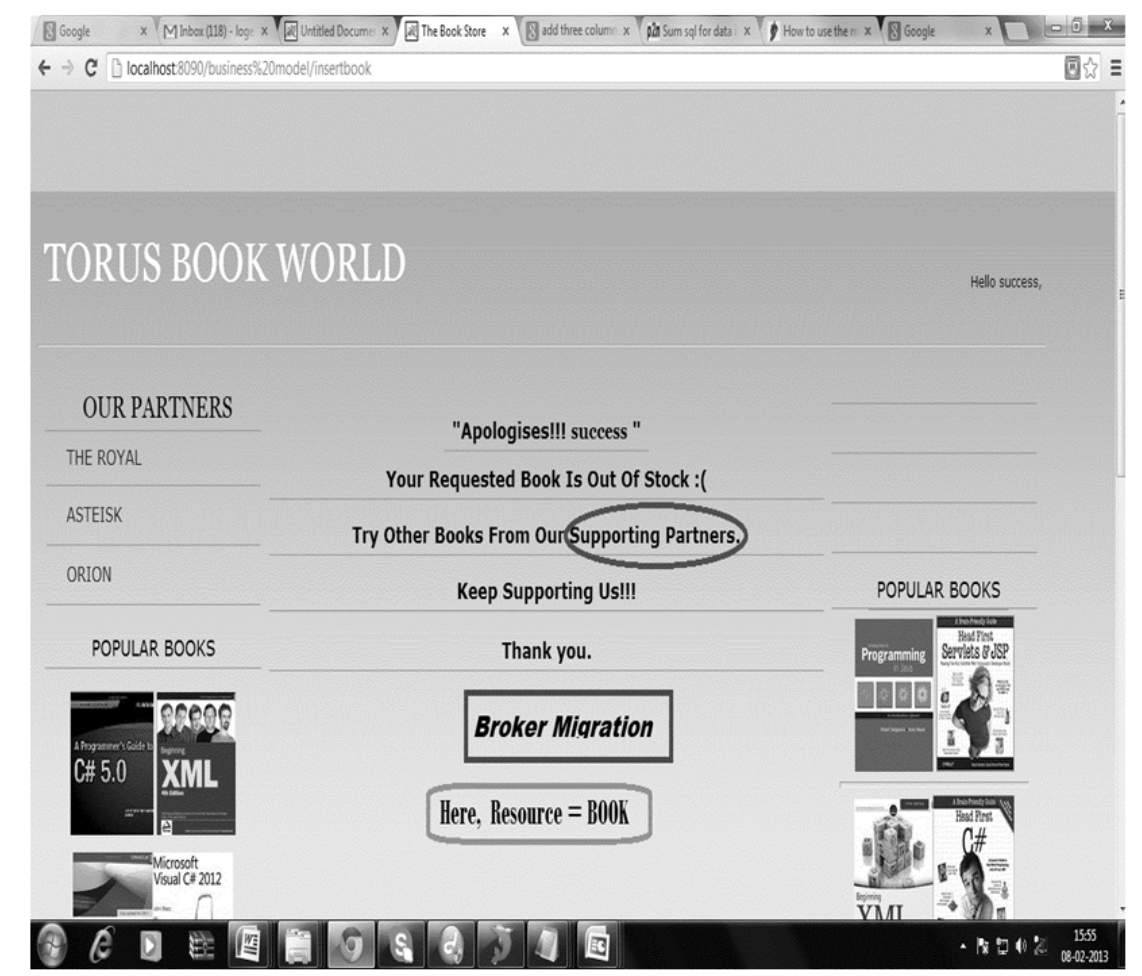

Fig. 5. Broker migration

\subsubsection{Algorithm}

Step 1: To begin with secured grid service at first have to login inside the service based on single sign on for different grid networks.

Step 2: At first suggestion based service request can be done. The broker can give valuable suggestion for the resource to the resource requesting users.

Step 3: If the resource is available in the particular in particular service they can purchase and just they can add to cart.

Step 4: If the particular product is not available in one service by job migration process it automatically skip to other service.

Step 5: The entire activity is carried out by grid brokers. Meta broker enables the users to access resources of different grids through their own brokers.

Step 6: Based upon the location the resource requisition can be done. Famous places got some famous resource so that it is the easy way of the resource requisition.

Step 7: The end of the lifetime the history of the purchased resource is very important for its service period.

\subsubsection{Experimental Result of Broker Migration}

In Fig. 5, if the available Product is not available in the particular service through job migration it skips to other supporting partner.

\subsection{Excellent Wireless Mobility Resource Access}

Access the wireless resources via resource providers, simultaneously from the grid portal for a job (Singh, 2011; Singh et al., 2013). Existing systems was not containing the inclusive disjunction model, so not efficient to get the cleaned data and normal data.

Propose an ORMT technique for proposed attackresistant, which is based on a dynamic, probabilistic model of the current attack situation.

In Equation 1, Distance Calculation algorithm:

Distance $=($ A / Quality $) *(1.92 / 2)$

Here:

A $\quad=$ rssi*rssi

rssi $=$ Received Signal Strength Indicator

Quality = Strength of Wi-Fi signal 


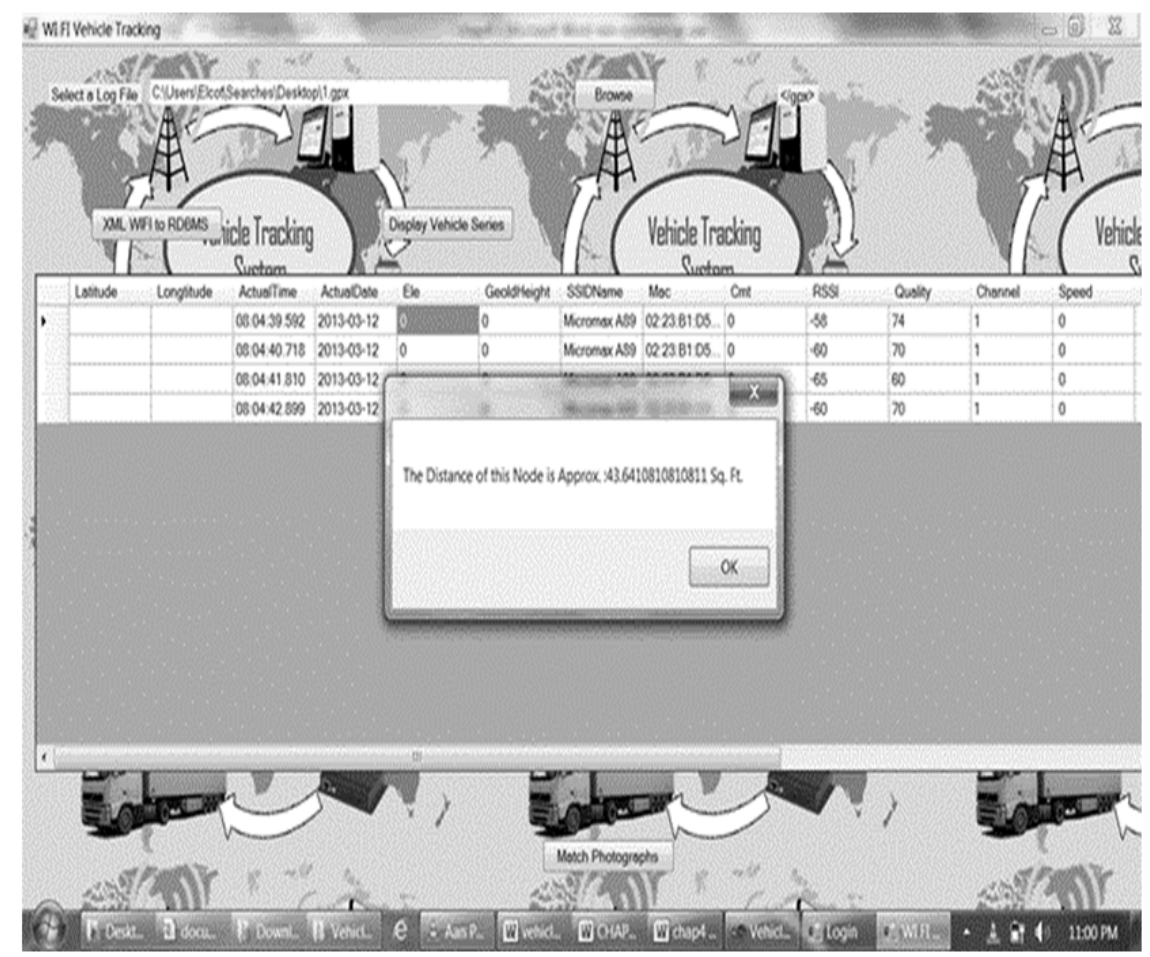

Fig. 6. Distance of the wireless mobility resource based on Wi-Fi/GPS grid services

In Equation 2, Location estimation algorithm:

$$
\mathrm{L}=\sum_{\mathrm{r}=1}^{\mathrm{R}} \mathrm{P}(\mathrm{Lr} \mid \mathrm{XV}) \cdot \mathrm{Lr}
$$

Here The algorithm:

$$
\begin{array}{ll}
\mathrm{r} & =\text { r-the reference location, } \\
\mathrm{R} & =\text { Reference locations, } \\
\mathrm{L} & =\text { The estimated location, } \\
\mathrm{P}(\mathrm{Lr} \mid \mathrm{XV}) & =\text { Probability of measuring } \mathrm{X}
\end{array}
$$

\subsubsection{Algorithm}

Step 1: Login Admin system.

Step 2: Get signal from Resources.

This is the process to get the tracking signal from resource. The signal may be Wi-Fi (or) GPS.

Step 3: Store the Resource Information.

The tracked information can be stored in .gpx format. In this format can be converted in to RDBMS for storing data in GRID database.

\section{Xml to RDMS File}

The user can be entering into the Wi-Fi user will log on to the services/then the user will use the services.
The input file in the form of $\mathrm{xml}$ files and the file can be clustered and the file in the form of RDMS format.

Step 4: Monitoring Source. $\log$ file.

Date and Time Calculation is made from the .gpx Step 5: Resource Security.

The Security of resource can be monitored from end user.

\subsubsection{Experimental of wireless mobility Resource Access}

In Fig. 6, gpx format file can contains like xml file and display traced mobility grid services with time and date. Calculate the node or resource distance.

\section{CONCLUSION}

This study is introduced an extensive level for Resource Management Technique (RMT). ORMTO use to reduce a cost, save a high-level energy, Increase the resource availability, Avoid the failure, Increased User satisfaction, easy handling for all levels of users, high Quality of Service (QoS), Reduce the Space and Time complexity in the grid network. This Paper can be 
providing a help to find a new research point in this field [ORMTO] in Future.

\section{ACKNOWLEDGEMENT}

The researchers would like to thank the reviewers for their detailed reviews and constructive comments, which have helped improve the quality of this paper. We would like to thank supported staff members for their comments on the study.

\section{REFERENCES}

Barzegar, B., H. Esmaeelzadeh and H. Shirgahi, 2011. A new method on resource management in grid computing systems based on QoS and semantics. Ind. J. Sci. Technol., 4: 1416-1419.

Bouyer, A. and M.N.M. Sap, 2010. A prediction-based fault tolerance on grid resources scheduling by using optimized case-based reasoning. University technology of Malaysia.

Dobre, C., R. Voicu and I. Legrand, 2011. Monitoring large scale network topologies. Proceedings of the IEEE 6th International Conference on Intelligent Data Acquisition and Advanced Computing Systems, Sept. 15-17, IEEE Xplore Press, Prague, pp: 218-222. DOI: 10.1109/IDAACS.2011.6072744

Folling, A., C. Grimme, J. Lepping and A. Papaspyrou, 2010. Robust load delegation in service grid environments. IEEE Trans. Parallel Distrib. Syst., 21: 1304-1316. DOI: 10.1109/TPDS.2010.16

Hu, L., X.L. Che and S.Q. Zheng, 2012. Online system for grid resource monitoring and machine learningbased prediction. IEEE Trans. Parallel Distrib. Syst., 23: 134-145. DOI: 10.1109/TPDS.2011.108

Javanmardi, S., S. Shaiatmadari, M. Mosleh and S.E.D.R. Abadi, 2013. A novel approach for grid resource management based on fuzzy logic and semantic technology. Int. J. Innovative Comput., 1: 23-31.

Netto, M.A.S. and R. Buyya, 2008. Rescheduling coallocation requests based on flexible advance reservations and processor remapping. Proceedings of the 9th Grid Computing Conference on Grid Computing, Sept. 29-Oct. 1, IEEE Xplore Press, pp: 144-151. DOI: 10.1109/GRID.2008.4662793
Nettoa, M.A.S., C. Vecchiolaa, M. Kirleya, C.A. Varelab and R. Buyya, 2011. Use of run time predictions for automatic co-allocation of multi-cluster resources for iterative parallel applications. J. Parallel Distrib. Comput. 71: 1388-1399.

Ning, X. and Y. Shaohua, 2013. A load-balanced crosspoint-queued switch fabric. China Commun., 10: 134-142. DOI: 10.1109/CC.2013.6472865

Ostberg, P.O. and E. Elmroth, 2013. GJMF-a composable service-oriented grid job management framework. Future Generat. Comput. Syst., 29: 144157. DOI: 10.1016/j.future.2012.04.004

Pradoa, R.B., F. Hoffmannb, S. Garcia-Galan, J.E.M. Expósitoa and T. Bertramb, 2012. On providing quality of service in grid computing through multiobjective swarm-based knowledge acquisition in fuzzy schedulers. Int. J. Approximate Reason., 53: 228-247. DOI: 10.1016/j.ijar.2011.10.005

Qiang, G., Z. Heng-Wei, W. Jing-Dong and W. Na, 2011. A grid resource matching algorithm, Proceedings of the International Conference on Computer Science and Network Technology, Dec. 24-26, IEEE Xplore Press, Harbin, pp: 142-145. DOI: 10.1109/ICCSNT.2011.6181927

Raj, R.J.S. and V. Vasudevan, 2011. Beyond simulated annealing in grid scheduling. Int. J. Inform. Sci. Comput., 5: 1-6.

Singh, A., 2011. An efficient load balancing algorithm for grid computing using mobile agent. Int. J. Eng. Sci., Technol., 3: 4744-4744.

Singh, S., M. Sarkar, S. Roy and N. Mukherjee, 2013. A survey on application of machine learning to resource management in grid environment. Lecture Notes Software Eng., 1: 173-177. DOI: 10.7763/LNSE.2013.V1.39

Surendran, R., B.P. Varthini, 2012. Integration based large scale broker's resource management on friendly shopping application in dynamic grid computing. Proceedings of the 4th International Conference on Advanced Computing, Dec. 13-15, IEEE Xplore Press, Chennai, pp: 1-6. DOI: 10.1109/ICoAC.2012.6416830 